\title{
Management of stage I occult uterine leiomyosarcoma: effects of morcellation, second-look surgery, and adjuvant treatments on survival
}

\author{
Jie Yang, Jiaxin Yang, Dongyan Cao, Lingya Pan, Ming Wu, Yang Xiang \\ Department of Obstetrics and Gynecology, Peking Union Medical College Hospital, Peking Union Medical College, Chinese Academy of Medical \\ Science, Beijing, China \\ Contributions: (I) Conception and design: Y Xiang; (II) Administrative support: Y Xiang; (III) Provision of study materials or patients: J Yang, D Cao, \\ L Pan, M Wu, Y Xiang; (IV) Collection and assembly of data: J Yang, Y Xiang; (V) Data analysis and interpretation: J Yang, Y Xiang; (VI) Manuscript \\ writing: All authors; (VII) Final approval of manuscript: All authors. \\ Correspondence to: Yang Xiang, MD. Department of Obstetrics and Gynecology, Peking Union Medical College Hospital, Peking Union \\ Medical College, Chinese Academy of Medical Science, No. 1. Shuaifuyuan, Wangfujing, Dong Cheng District, Beijing 100730, China. \\ Email: xiangy@pumch.cn.
}

Background: This study sought to analyze the risk of morcellation in patients who underwent surgery for leiomyoma and had a final pathological diagnosis of uterine leiomyosarcoma (uLMS), and evaluate the survival benefits of second-look surgery and chemotherapy in patients with stage I occult uLMS.

Methods: A retrospective analysis of the data of patients with occult stage I uLMS in the Peking Union Medical College Hospital database between 2005 and 2018 was conducted. The recurrence rate and progression-free survival (PFS) were compared between patients who underwent morcellation or not. Univariate analyses were used to evaluate the survival impact of lymphadenectomy, oophorectomy and adjuvant chemotherapy. Propensity-score matching methods were used to evaluate the effect of morcellation on recurrence while adjusting for baseline confounding factors using Poisson regression fitted by inverse probability weighting (IPW) estimation.

Results: A total of 96 patients with uLMS were identified among the 31,679 surgeries performed for leiomyomas (incidence: $0.303 \%$ ). Hysterectomy was performed in 60 patients, and myomectomy was performed in 36 patients (power morcellation $\mathrm{n}=20)$. There were $36(37.5 \%)$ patients underwent lymphadenectomy, and 76 (79.2\%) patients underwent oophorectomy. Among them, 47 (52.8\%) patients received postoperative chemotherapy. The median follow-up time was 40 months (range, 12-146 months), and there were 43 cases of recurrence $(44.7 \%)$. No differences in recurrence were found between the hysterectomy and myomectomy groups (hazard ratio $0.839, \mathrm{P}=0.701$ ). The 3 -year PFS rates for patients with hysterectomy, power morcellation, and non-power morcellation were $64.3 \%, 53.8 \%$, and $59.8 \%$, respectively. No survival differences were identified between patients with/without lymphadenectomy [PFS: $\mathrm{P}=0.513$; overall survival $(\mathrm{OS})$ : $\mathrm{P}=0.413$ ] and oophorectomy ( $\mathrm{PFS}: \mathrm{P}=0.162$; OS: $\mathrm{P}=0.815$ ). Postoperative chemotherapy was associated with better PFS $(\mathrm{P}=0.047)$, but not OS $(\mathrm{P}=0.36)$.

Conclusions: No survival differences were observed among the initial surgical procedures in stage I patients with occult uLMS. No survival benefits were observed between lymphadenectomy and oophorectomy patients. Compared to continued observation, postoperative chemotherapy was associated with improved PFS, but not OS.

Keywords: Occult; uterine leiomyosarcoma (uLMS); morcellation; second-look surgery; chemotherapy.

Submitted Nov 09, 2021. Accepted for publication Dec 22, 2021.

doi: 10.21037/atm-21-6424

View this article at: https://dx.doi.org/10.21037/atm-21-6424 


\section{Introduction}

Uterine sarcoma is a rare tumor that accounts for $<1 \%$ of female malignancies and $2-5 \%$ of all malignant uterine tumors (1). Uterine leiomyosarcoma (uLMS) accounts for $30 \%$ of all uterine sarcomas, and is often diagnosed after surgery for presumed leiomyoma (2). The incidence of occult uLMS ranges from 0.2 to 2.33 per 1,000 hysterectomies $(3,4)$. It is difficult to differentiate sarcoma and benign myoma without pathological test. It is reported obesity, history of diabetes and advanced age are associated with uterine sarcoma (2). However, none of these features are sufficient for pre-surgical diagnosis. Thus, it is inevitable for patients who underwent surgery for benign uterine leiomyoma are diagnosed with uterine sarcoma post operation. Myomectomy and hysterectomy are the main surgical managements for uterine myoma, and morcellation is a common approach especially in minimally invasive surgery. There is increasing concern that myomectomy and power morcellation can lead to the peritoneal dissemination of occult uLMS. Some studies have suggested that the use of morcellation in patients with occult uLMS who have undergone a hysterectomy has a detrimental effect on survival (3,5-7). However, population-based studies have been unable to accurately assess associations between power morcellation and survival due to their small numbers (3). To date, most studies have been retrospective small-series studies on heterogeneous morcellation techniques (7), and the effects of morcellation on survival have not been fully evaluated.

In the management of patients with LMS confined to the uterus, hysterectomy with or without bilateral salpingooophorectomy (BSO) is recommended and adjuvant chemotherapy is not suggested (8). Many retrospective studies have reported a low incidence of ovarian and pelvic lymph node metastasis in uLMS (9-11). Lymph node metastasis is reported in $<3 \%$ of uLMS patients with disease limited to the uterus $(9,10)$. The incidence of ovarian metastasis ranges from $3.5 \%$ to $4 \%$ (11). Due to the rarity and the difficulties of preoperative diagnosis, there is a lack of evidence on the surgical management of, and use of adjuvant therapy in, patients with occult uLMS diagnosed after undergoing a myomectomy or hysterectomy for leiomyomas. For patients who had hysterectomy at the initial surgery, some physicians may prefer the imaging evaluation for extra-uterine disease rather than secondlook surgery. There is no consensus on the use of secondlook surgery with oophorectomy or lymphadenectomy, especially in patients with a tumor confined to uterus who underwent a hysterectomy. The value of re-exploration with lymphadenectomy and/or oophorectomy are poorly defined and controversial.

Since the management of stage I occult uLMS is not fully elucidate, we retrospectively reviewed the details of the initial surgery and management after diagnosis of uLMS patients who underwent operations to treat leiomyoma at one academic center. This study sought to evaluate the survival outcomes of occult uLMS patients undergoing different primary surgeries and evaluate the benefits of second-look surgery with lymphadenectomy and/or oophorectomy, and adjuvant chemotherapy.

We present the following article in accordance with the STROBE reporting checklist (available at https://atm. amegroups.com/article/view/10.21037/atm-21-6424/rc).

\section{Methods}

The study was conducted in accordance with the Declaration of Helsinki (as revised in 2013). The Institutional Review Board of the Peking Union Medical College Hospital approved this retrospective study (No. S-K1559) and individual consent for this retrospective analysis was waived. We reviewed the clinical records of patients with stage I uLMS who underwent surgery for leiomyoma from 2005 to 2018 at our institution. The following information was extracted from the medical records: age at time of cancer diagnosis, clinical manifestation, tumor stage [2009 International Federation of Gynecology and Obstetrics (FIGO) staging], pathologic tumor size (largest diameter), grade (well differentiated, moderately differentiated, poorly differentiated, or undifferentiated), mitotic count ( $\leq 15$ or $>15$ mitotic count/10 high-power field (HPF), and necrosis.

The initial surgery for leiomyoma included open or laparoscopic myomectomy and hysterectomy. At our institution, open myomectomy was performed uncontained and specimens were manually fragmented with a scalpel or scissors in the peritoneal cavity. In the laparoscopic myomectomy, power morcellation was performed in the peritoneal cavity or in-bag. Specimens from the laparoscopic hysterectomy were extracted uncontained from the vagina with or without scalpel morcellation in the vagina. After a diagnosis of uLMS in patients who underwent a myomectomy, a subsequent hysterectomy was performed with or without lymphadenectomy and/or oophorectomy. The decision to proceed with lymphadenectomy in secondlook surgery was made by the gynecologic oncologist 
according to imaging and intraoperative findings. If there was extra-uterine tumor spread or suspicious lymph nodes, lymphadenectomy was performed. Surgical lymph node dissection (LND) was performed via laparotomy or laparoscopy, including in areas of the common, external, internal iliac artery, and the obturator fossa. BSO was performed on postmenopausal women and was optional in premenopausal women based on shared decision making between the physician and patient. Perioperative complications of Clavien-Dindo (12) grade III-IV occurring from day 1 to 30 after surgery were documented.

The decision for adjuvant treatment was made by a multidisciplinary team that included a gynecologic oncologist, radiation oncologist, and medical oncologist. Chemotherapy regimens included doxorubicin-based therapy, such as doxorubicin plus cisplatin plus ifosfamide, doxorubicin plus cisplatin, and gemcitabine plus docetaxel. No adjuvant radiation therapy was administered to any patient in this study because only stage I disease patients were included.

After treatment completion, patients were followed every 3-6 months for the first 2 years, 6-12 months for 3-5 years, and annually thereafter. Recurrence was confirmed by either imaging or pathologic examination after surgical resection. The pattern of recurrence was documented as peritoneal (vaginal cuff, pelvis, or abdomen), lymph node metastasis (retroperitoneal, or above para-aortic nodes), hematogenous (liver, lung, bone, or brain) and multiple. Progression-free survival (PFS) was defined as the time (months) from initial diagnosis to disease recurrence. Overall survival (OS) was defined as the time (months) from diagnosis to death from all causes. Data on patients with no evidence of disease recurrence or death were censored at the date of the last follow up.

\section{Statistical analysis}

The Mann-Whitney $\mathrm{U}$ test was used to analyze continuous variables as appropriate. Frequency distributions were compared using Chi-square and Fisher's exact test for categorical variables. Propensity-score matching methods were used to evaluate the effect of morcellation on recurrence while adjusting for baseline confounding factors using poisson regression fitted by inverse probability weighting (IPW) estimation. We estimated the propensity scores using logistic regression models with the covariates of age ( $\leq 50$ or $>50$ years), stage (IA, tumor size $\leq 5 \mathrm{~cm}$ or IB, $>5 \mathrm{~cm}$ ), grade (well and moderately differentiated, or poorly differentiated and undifferentiated), mitotic count ( $\leq 15$ or $>15 / 10 \mathrm{HPF}$ ), oophorectomy (yes or no), LND (yes or no), morcellation at initial surgery (yes or no), and adjuvant chemotherapy (yes or no).

Survival outcomes (PFS and OS) were estimated using the Kaplan-Meier method and log-rank test. Cox proportional hazards ratio (HR) analyses were performed to identify independent prognostic factors. Predictors for survival (i.e., age, initial surgery type, stage, tumor grade, mitotic counts, and adjuvant chemotherapy) were assessed by univariate analyses. Multivariate Cox models included factors associated with a statistically significant increased risk for recurrence or death in the univariate analysis. In all cases, a $\mathrm{P}$ value $<0.05$ was considered statistically significant. SPSS ver. 23.0 (IBM, Armonk, NY, USA) and Prism 6.0c software (GraphPad, San Diego, CA, USA) were used for the statistical analyses.

\section{Results}

\section{Demographic features}

From January 2005 to December 2018, 31,679 patients underwent a myomectomy or hysterectomy for leiomyoma at the Gynecologic Department. We identified 118 patients with a final diagnosis of uLMS. Among them, 22 patients had advanced disease with either an extra-uterine tumor or bulky lymph node metastasis. Comprehensive debulking surgery was performed at the initial surgery after a pathological diagnosis of uLMS from a frozen section. The other 96 patients with a tumor confined to the uterus were diagnosed postoperatively and included in this study. The incidence of stage I occult uLMS among women who underwent surgery for leiomyoma was $0.303 \%$.

The patients had a mean age of 45.9 years (range, $22-76$ years). The majority of patients $(n=40,41.7 \%)$ had insignificant symptoms, such as abdominal bulging and bloating, which were diagnosed with uterine leiomyoma on imaging at an annual health visit. The surgical indication for these patients without significant symptoms was the large size of the fibroid. Other symptoms included increasing uterine "leiomyoma" size ( $\mathrm{n}=27,28.1 \%)$, abnormal uterine bleeding ( $\mathrm{n}=21,21.9 \%)$, abdominal pain $(\mathrm{n}=7,7.3 \%)$, and urinary retention $(\mathrm{n}=1,1.0 \%)$. The average tumor maximum diameter was $7.7 \mathrm{~cm}$ [standard deviation (SD) $3.3 \mathrm{~cm}$; range, $1-20 \mathrm{~cm}$ ]. Tumor grade was divided into well differentiated/moderately differentiated $(n=16,16.7 \%)$ and poorly differentiated/undifferentiated $(n=80,83.3 \%)$ 
Table 1 Patient demographics and tumor characteristics by initial surgery subgroup

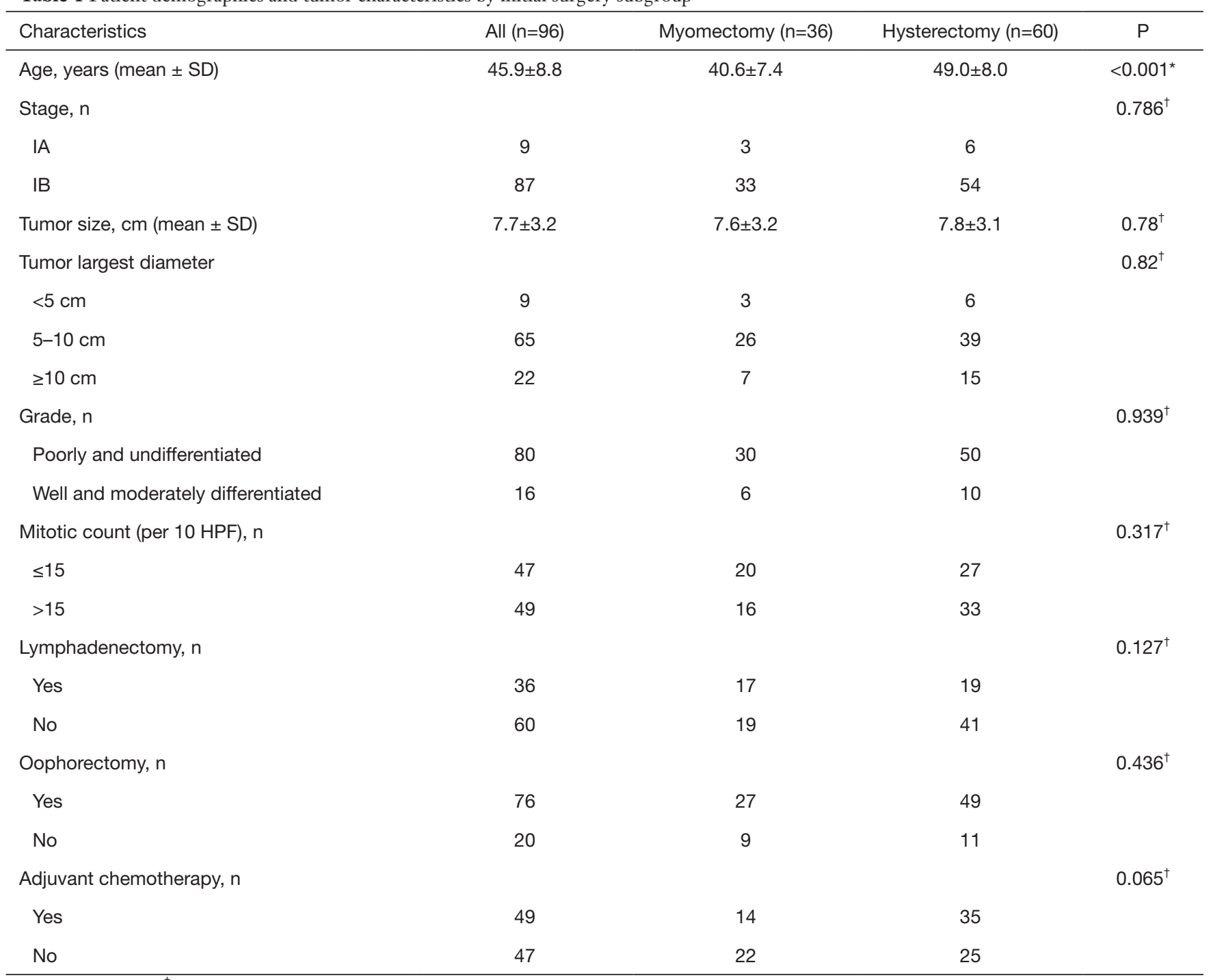

${ }^{*}$, Student $t$-test; ${ }^{\dagger}$, Chi-square test. SD, standard deviation; HPF, high-power field.

category. Table 1 summarizes patient demographics and tumor characteristics by initial surgery subgroup.

\section{Surgical management}

In relation to the initial surgery, 60 patients underwent a hysterectomy, and 36 patients underwent a myomectomy (16 open and 20 laparoscopic). In-bag power morcellation was performed in 2 patients $(2 / 20,10 \%)$ after the year 2017. Secondary surgery was performed in 62 patients with a median time of 30 days from the initial surgery (range, 7-73 days). The median waiting time from myomectomy to hysterectomy was 25 days (range, 7-64 days). A total of 36 patients underwent LND, and 76 patients received oophorectomy. One intraoperative complication of bladder injury was identified in a patient during secondary LND. 49 patients who received adjuvant chemotherapy. The surgical treatment is illustrated in Figure 1.

\section{Follow-up}

The median follow-up time was 41 months (range, 12-146 months). Overall, there were 43 recurrences (44.8\%) with a median recurrence time of 33 months (range, 5-119 months). The analysis of the relapse pattern revealed that $8(18.6 \%)$ patients had recurrence in the vaginal cuff, 


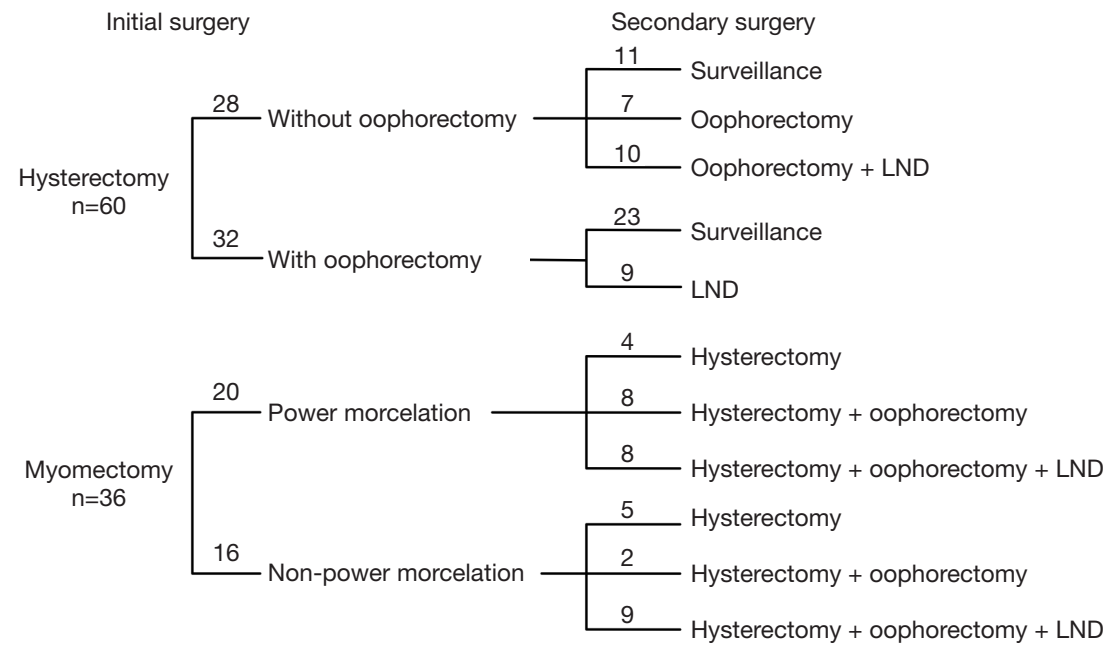

Figure 1 Treatment algorithm. LND, lymphadenectomy.

Table 2 Recurrence pattern by initial surgery subgroup

\begin{tabular}{|c|c|c|c|c|c|}
\hline Recurrence sites & All $(n=96)$ & \multicolumn{2}{|c|}{ Myomectomy } & \multicolumn{2}{|c|}{ Hysterectomy } \\
\hline Total (n) & 43 & 9 & 6 & 22 & 6 \\
\hline \multicolumn{6}{|l|}{ Peritoneal, n (\%) } \\
\hline Vaginal cuff & $8(18.6)$ & $1(11.1)$ & $1(16.7)$ & $3(13.6)$ & $3(50.0)$ \\
\hline Abdomen & $8(18.6)$ & $4(44.4)$ & $0(0.0)$ & $4(18.2)$ & $0(0.0)$ \\
\hline Lymph node, n (\%) & $2(4.7)$ & $1(11.1)$ & $1(16.7)$ & $0(0.0)$ & $0(0.0)$ \\
\hline Hematogenous, n (\%) & $4(9.3)$ & $1(11.1)$ & $1(16.7)$ & $2(9.1)$ & $0(0.0)$ \\
\hline Multiple, n (\%) & $6(13.0)$ & $1(11.1)$ & $1(16.7)$ & $3(6.4)$ & $1(16.7)$ \\
\hline
\end{tabular}

$15(34.9 \%)$ in the pelvis, $8(18.6 \%)$ in the abdomen peritoneum, 2 (4.7\%) had retroperitoneal pelvic lymph node metastasis only, $4(9.3 \%)$ had a hematogenous pattern only, and $6(13.0 \%)$ had multiple metastases (Table 2). We observed a high recurrence rate in the abdomen and peritoneum in patients who underwent power morcellation (44.4\%), and vaginal cuff relapse in patients who underwent laparoscopic hysterectomy with vaginal fragmentation of the specimen (50\%). There was 1 of the 2 patients who received contained morcellation during laparoscopic myomectomy had a relapse in the pelvis and lung metastasis 17 months after the first surgery. Of the 21 patients whose ovaries were preserved in the initial treatment, 13 patients relapsed and 9 had ovarian involvement $(9 / 13,69.2 \%)$. In these patients, the ovaries were not the only site of recurrence.

\section{Survival outcomes}

Under the IPW model, which adjusted for the confounding factors of age, tumor stage, grade, mitotic count, LND, oophorectomy, and adjuvant chemotherapy, no differences were found in recurrence between the hysterectomy and myomectomy groups in the initial surgery [HR 0.839 , 95\% confidence interval (CI): $0.343-2.054, \mathrm{P}=0.701]$. In the survival analyses, the 3 -year PFS rate was $64.3 \%$ for the hysterectomy group and $55.7 \%$ for the myomectomy group (HR 0.98, 95\% CI: 0.52-1.84, $\mathrm{P}=0.945$ ). The 3-year OS was $92.1 \%$ for the hysterectomy group and $95.0 \%$ for the myomectomy group (HR 2.58, 95\% CI: 0.68-9.81, $\mathrm{P}=0.163)$. In analyzing the effect of power morcellation, the 3 -year PFS was $53.8 \%$ for the power-morcellation 
group and $59.8 \%$ for the non-power morcellation group (HR 1.18, 95\% CI: $0.45-3.27, \mathrm{P}=0.751$ ). We found no survival differences in patients who underwent secondary LND in relation to PFS (HR 0.81, 95\% CI: 0.43-1.51, $\mathrm{P}=0.513$ ) and $\mathrm{OS}$ (HR 1.63, 95\% CI: 0.47-5.69, $\mathrm{P}=0.413$ ) and those who only underwent a hysterectomy. The 3-year PFS in patients who received an oophorectomy was $65.6 \%$, as compared to $45.7 \%$ in patients who did not (HR 0.64, 95\% CI: 0.31-1.32, $\mathrm{P}=0.162)$. We observed no differences in the OS of patients with or without oophorectomy (HR 0.86, 95\% CI: 0.22-3.39, P=0.815) (Figure 2). Patients who received adjuvant chemotherapy had better PFS (HR 0.54, 95\% CI: 0.29-0.99, P=0.047), but not OS (HR $0.57,95 \%$ CI: $0.17-1.86, \mathrm{P}=0.36)$ than patients who did not receive chemotherapy (Figure 3). The results of the univariate analysis of PFS are shown in Table 3. Histologic grade (HR 0.46, 95\% CI: $0.23-0.79, \mathrm{P}=0.034$ ) was another factor associated with PFS. Tumor grade, mitotic count, oophorectomy, and adjuvant chemotherapy were examined in the multivariate analysis of PFS, but none of these factors had a significant effect on survival (Table 3).

\section{Discussion}

This study focused on patients with stage I occult uLMS who underwent a hysterectomy or myomectomy to treat uterine leiomyoma. The recurrence rate was high, and the median time to recurrence was 33 months. We found no survival differences based on the type of initial surgery, but the pattern of recurrence differed slightly. Secondary lymphadenectomy and oophorectomy were not associated with additional survival benefits. Patients who underwent adjuvant chemotherapy had better PFS than those who did not undergo adjuvant chemotherapy.

It is difficult to diagnose uLMS clinically without a surgical-pathological assessment. Presurgical imaging methods cannot be used to discriminate between sarcomas and benign leiomyomas when the tumor is confined to the uterus. A curettage biopsy may be helpful, but it has less sensitivity in diagnosing uLMS than endometrial cancers (8). Over $50 \%$ of stage I uterine sarcomas are occult, and the incidence of uLMS among women who have undergone hysterectomies for leiomyomas is about $0.2-2.33$ per 1,000 $(3,4,13)$. In this institutional study, the incidence of stage I uLMS in patients who underwent a hysterectomy or myomectomy for presumed uterine leiomyoma was 3.03 per 1,000 , a figure slightly higher than that previously reported. Our incidence rate may have been overestimated due to a selection bias error related to our hospital being a tertiary referral center, and our decision not to include women who underwent hysterectomies with leiomyomas listed as a secondary diagnosis on their discharge records.

\section{Effects of morcellation on survival}

Primary hysterectomy without any kind of tumor fragmentation is considered the most appropriate treatment for early stage uLMS. Tumor fragmentation may change the recurrence pattern and natural history of the early stage disease (14-17). Despite adjusting the prognosis predictors and minimizing the bias of the heterogeneity of the data, several population-based studies have reported different results $(3,18)$. A large population-based cohort study of 111 stage I occult uLMS patients showed that none of the adjusted risk ratios for the recurrence or death were significant for 3 -year survival in patients with no morcellation, power, and non-power morcellation (3). The 3-year PFS rates for stage I leiomyosarcomas with no morcellation, power morcellation, and non-power morcellation were $54 \%, 19 \%$, and $51 \%$, respectively (3). Similarly, in the present study, the 3 -year PFS rates for hysterectomy, power morcellation, and non-power morcellation were $64.3 \%, 53.8 \%$, and $59.8 \%$, respectively. Another study of the New York State Cancer Registry and database identified 231 women who had occult uLMS (18). This propensity-score adjusted study showed that patients who underwent power morcellation had a higher mortality rate than those who underwent abdominal supracervical hysterectomy (HR 3.64, 95\% CI: 1.5-8.86) and total abdominal hysterectomy (HR 4.66, 95\% CI: 1.97-11.0). However, no significant differences were observed for allcause mortality. Notably, only 21 patients received power morcellation in this study (18).

In a meta-analysis of 202 patients with stage I-III unexpected uLMS, 75 patients had morcellation in the initial surgery (19). This pooled data suggests a significant correlation between morcellation and increased intraabdominal recurrence (OR 4.11, 95\% CI: 1.92-8.81) and death (OR 2.42, 95\% CI: 1.19-2.42) (19). In another metaanalysis of 715 women with uLMS, 384 had morcellation (13). The 5 -year survival estimate for power morcellation (30\%) was lower than that for scalpel morcellation (59\%) and no morcellation (60\%), but as the results were significant, the detrimental effect of power morcellation was not proven.

In our study, the surgical procedures were highly consistent. In the propensity score-based IPW model, we 

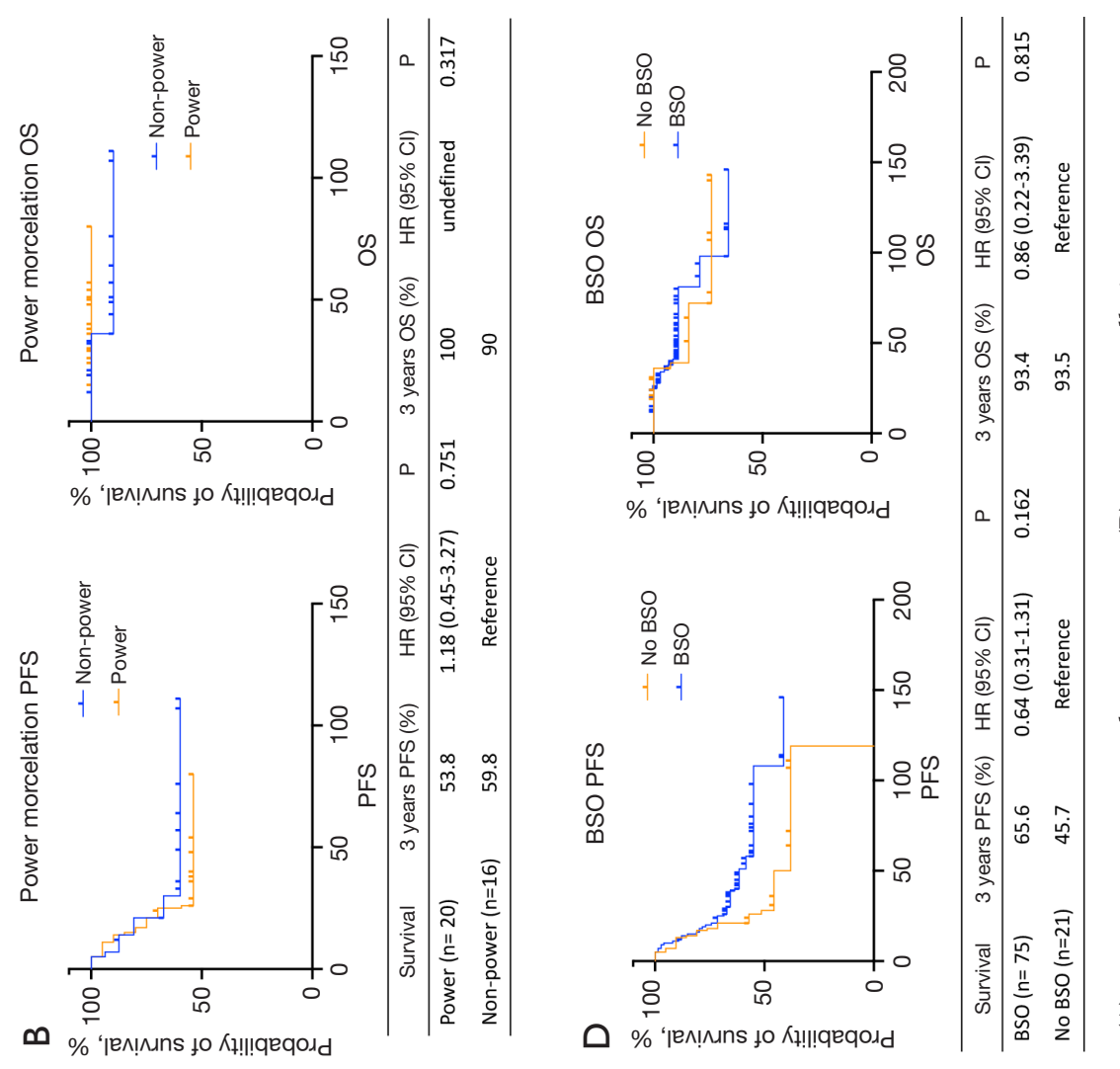

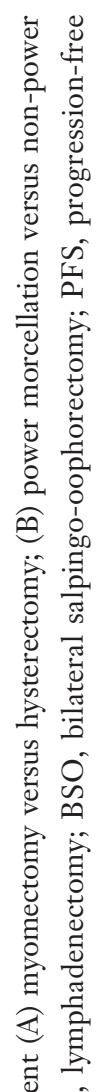
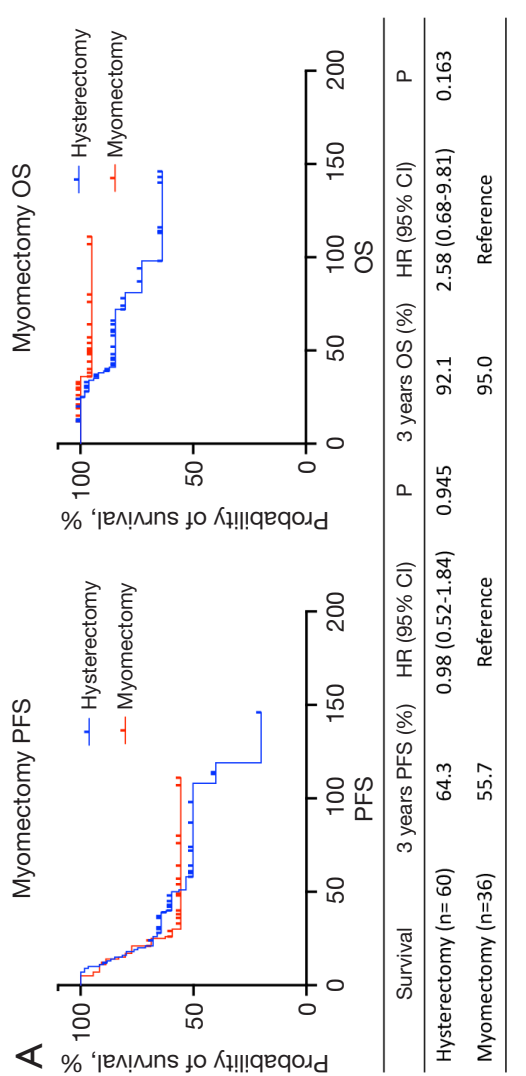

\% 'ien!mins to Kt!!!queqod
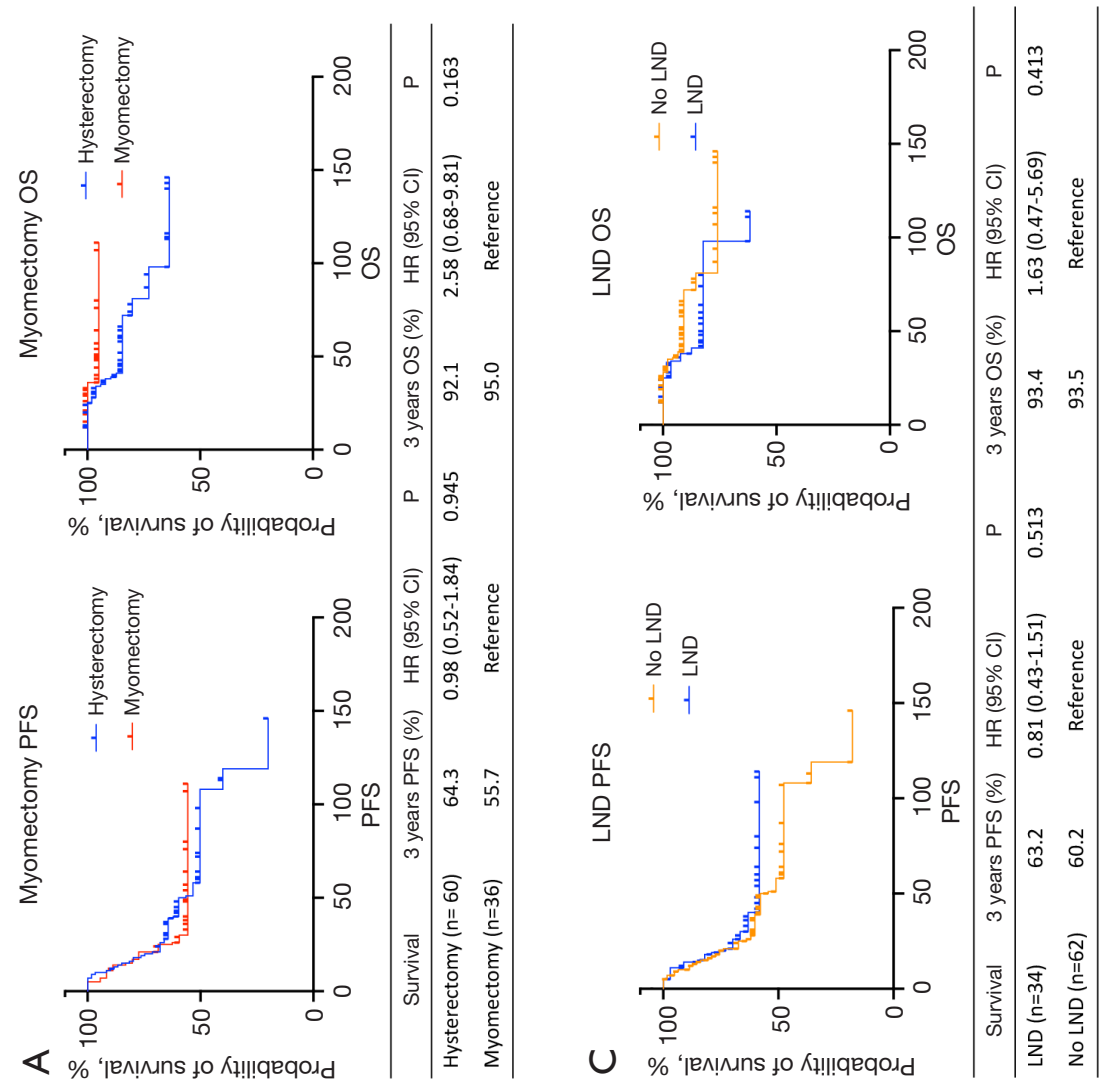

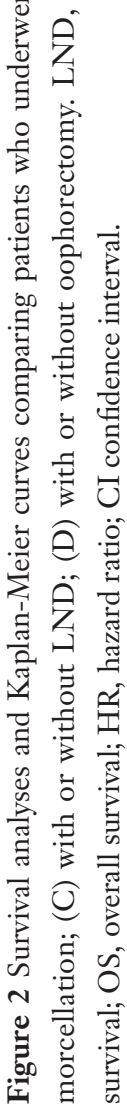



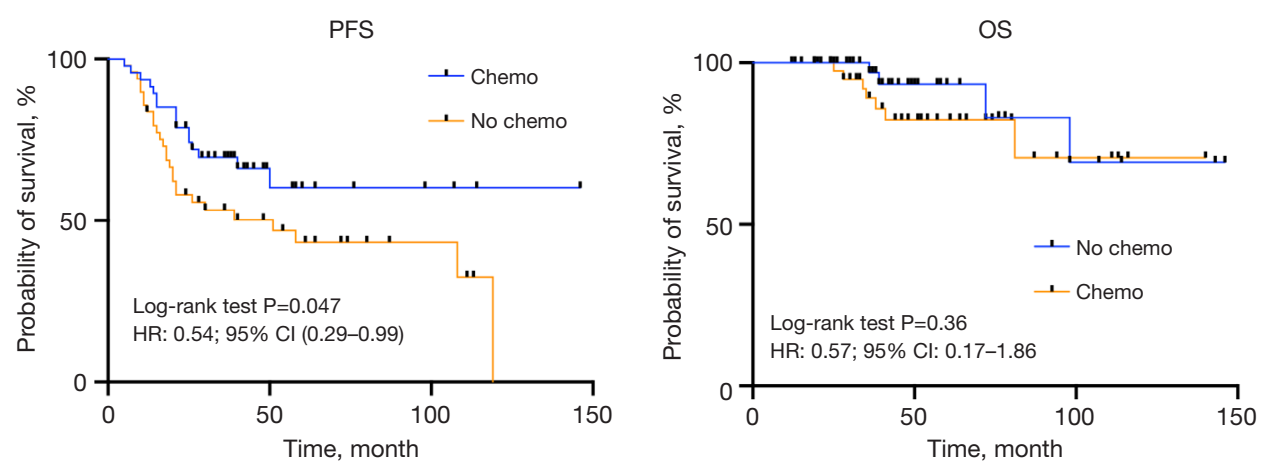

Figure 3 Survival analyses and Kaplan-Meier curves comparing patients with or without adjuvant chemotherapy. PFS, progression-free survival; OS, overall survival; HR, hazard ratio; CI, confidence interval.

did not find any differences in the recurrence rates between the myomectomy and hysterectomy groups. However, there was a higher intraperitoneal relapse in the powermorcellation group (44.4\%) and local relapse in patients who underwent vaginal morcellation $(50.0 \%)$. Thus, tumor fragmentation may change the pattern of recurrence without affecting OS outcomes. Further, contained morcellation may help prevent tumor spillage, but this requires further evaluation.

The similar survival rates between patients who receive morcellation and an en bloc removal of the tumor may be due to the aggressive malignant behavior of uLMS, which can spread rapidly through hematogenous pathways. Even when the tumor was confined to the uterus, the 5 -year OS rate was only $55.4 \%$ (20). The inadvertent rupture of the uterus during hysterectomy may also lead to tumor spillage and intra-abdomen implantation. Based on existing data, The American College of Obstetricians and Gynecologists recommend a minimally invasive approach for younger women, and no morcellation in postmenopausal women (4).

\section{Second-look surgery}

A second-look surgery with hysterectomy is necessary in patients who undergo a myomectomy and are unexpectedly diagnosed with uLMS. However, systemic staging surgery with an oophorectomy and LND is controversial in stage I uLMS (8). There is a consensus that ovaries should be removed in postmenopausal women (21). Most studies have found that ovarian preservation is safe in stage I premenopausal women, who have similar relapse and survival rates to women who undergo BSO (22). In a Surveillance, Epidemiology, and End Results (SEER) case series study, no difference was found in mortality even after controlling for age and FIGO substage (23). A National Cancer Database study of 7,455 patients suggested that oophorectomy, which is not associated with survival, should be omitted in women aged $\leq 51$ years at diagnosis (20). In our series, we also found no survival benefits associated with oophorectomy.

Of the patients who preserved ovaries at the initial management, $69.2 \%$ had ovarian involvement at recurrence, but the ovaries were not the only site of recurrence. In our view, the decision to perform an oophorectomy should be balanced against the patient's age, quality of life, and survival benefits. In premenopausal patients, a shared decision should be made by both the clinician and the patient, after explaining the risks and benefits of ovarian preservation.

The incidence of lymph node metastasis is reported to range from $6 \%$ to $26 \%$ in patients with lymph node metastasis who had at least clinical stage II disease $(6,10,11)$ and the majority of patients $(87.5 \%)$ who had extra-uterine spread (10). The role of lymphadenectomy in stage I uLMS is controversial (24). Some studies have found that lymphadenectomy has a therapeutic effect in uterine sarcomas, especially in patients with extra-uterine diseases $(25,26)$. However, these studies included all types of uterine sarcoma. A meta-analysis of $4867 \mathrm{uLMS}$ patients (1,356 of whom underwent lymphadenectomy) suggested that there was a lack of evidence of the survival benefits of lymphadenectomy (24). In this case series, none of the patients with tumors confined to the uterus had lymph node metastasis, which is consistent with other studies that found that the lymph node metastatic rate is $<3 \%$ in early stage uLMS (10). We found no differences in recurrence or 3-year survival outcomes in patients who underwent lymphadenectomy rather than surveillance. Additionally, the lymph node metastatic rate at recurrence was also low $(2 / 43$, 
Table 3 Univariate and multivariate analysis of prognostic factors in PFS

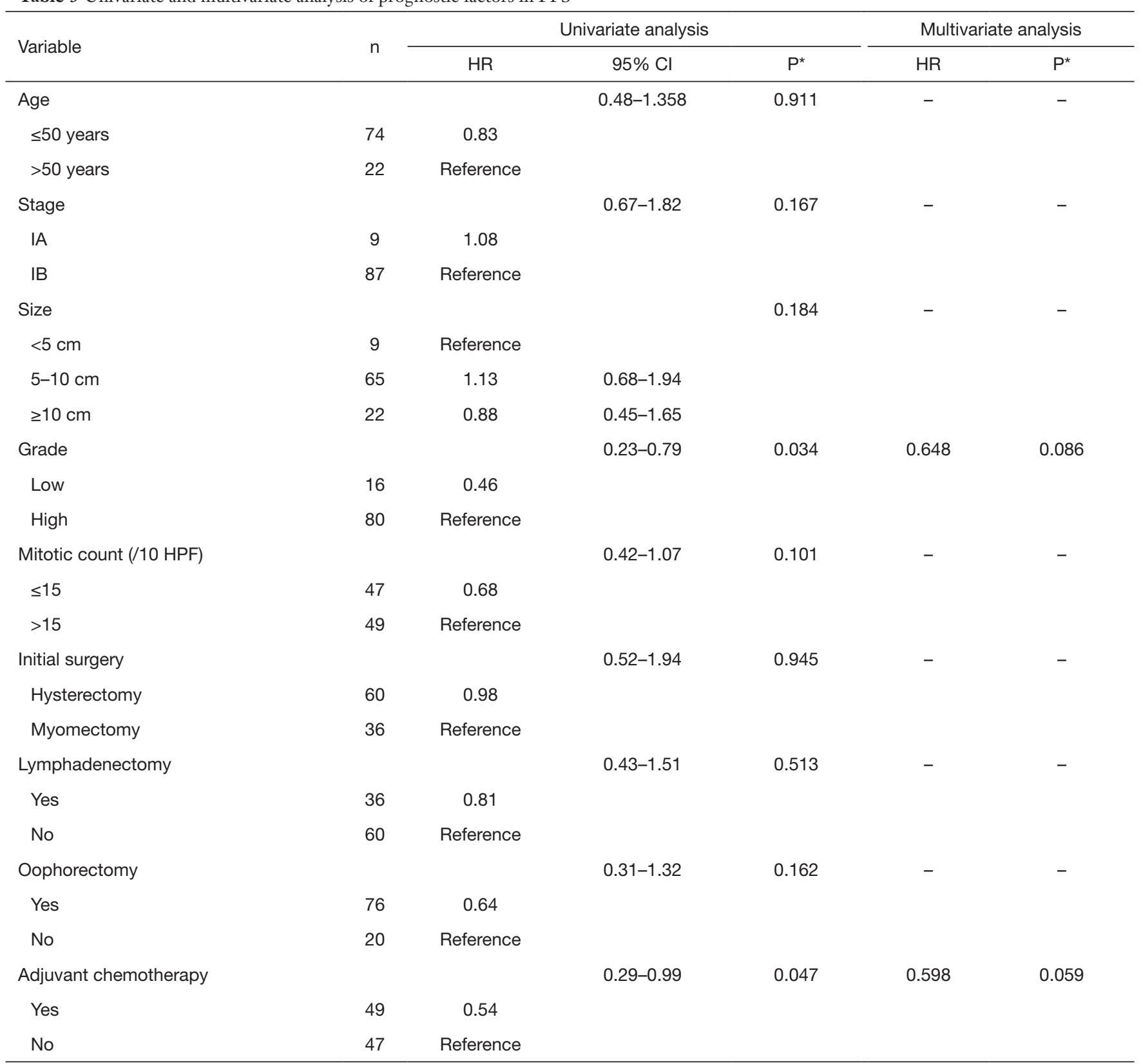

*, log-rank test. PFS, progression-free survival; HR, hazard ratio; $\mathrm{Cl}$, confidence interval; HPF, high-power field.

96\%). We suggest routine LND should not be performed at the second-look surgery in patients with uLMS confined to the uterus.

\section{Adjuvant treatment}

The therapeutic effect of systemic chemotherapy is poorly defined for patients with uLMS. A recent study in
Korea of 55 patients with stage I occult uLMS found that adjuvant treatment produced no improvement in survival outcomes (27). However, this small cohort study was heterogeneous with 3 kinds of morcellation techniques (intra-abdominal power morcellation, scalpel, or vaginal) and both chemotherapy and radiation were included as the adjuvant treatment. An ongoing phase III trial (GOG 277) is assessing the role of postoperative adjuvant chemotherapy 
versus observation in patients with high-grade stage I and II uLMS (28). As far we recommend adjuvant chemotherapy in management of occult uLMS.

The role of adjuvant radiotherapy in stage I uLMS is controversial. A phase III trial of stage I and II uLMS reported that postoperative pelvic radiotherapy did not improve OS more than observation (29). Most retrospective studies of adjuvant radiation therapy suggest an improvement in local pelvic control but no appreciable or consistent improvement in OS due to the propensity of metastatic extra-pelvic disease as the first or eventual recurrence (8). Routine adjuvant radiation is not recommended for stage I patients with uLMS in the National Comprehensive Cancer Network guidelines. In our series of patients with vaginal morcellation, $52 \%$ had solitude vaginal cuff recurrence. Such patients may benefit from radiation therapy to control local recurrence.

Our study had several limitations. First, it was a retrospective study and thus has unavoidable inherent biases. Second, the treatments were heterogeneous according to patient status and physician preferences. However, these concerns do not change the conclusions of our primary outcome of interest. Our study also had a number of strengths. First, the data were collected from one academic medical institution and the surgical procedures were highly homogenous. Second, a propensity-score weighting model was used to adjust for the differences between groups without discarding patients.

In conclusion, we observed no difference in survival outcomes between stage I uLMS patients who underwent a myomectomy or hysterectomy as the initial surgery for leiomyoma, but morcellation may change the recurrence pattern. We did not find any survival benefits associated with a secondary lymphadenectomy or oophorectomy. Adjuvant chemotherapy was associated with better PFS, but not OS, than observation.

\section{Acknowledgments}

Funding: None.

\section{Footnote}

Provenance and Peer Review: This article was commissioned by the Guest Editor (Danbo Wang) for the series "New Progress and Challenge in Gynecological Cancer" published in Annals of Translational Medicine. The article has undergone external peer review.
Reporting Checklist: The authors have completed the STROBE reporting checklist. Available at https://atm. amegroups.com/article/view/10.21037/atm-21-6424/rc

Data Sharing Statement: Available at https://atm.amegroups. com/article/view/10.21037/atm-21-6424/dss

Conflicts of Interest: All authors have completed the ICMJE uniform disclosure form (available at https://atm. amegroups.com/article/view/10.21037/atm-21-6424/coif). The series "New Progress and Challenge in Gynecological Cancer" was commissioned by the editorial office without any funding or sponsorship. The authors have no other conflicts of interest to declare.

Etbical Statement: The authors are accountable for all aspects of the work in ensuring that questions related to the accuracy or integrity of any part of the work are appropriately investigated and resolved. The study was conducted in accordance with the Declaration of Helsinki (as revised in 2013). The Institutional Review Board of the Peking Union Medical College Hospital approved this retrospective study (No. S-K1559) and individual consent for this retrospective analysis was waived.

Open Access Statement: This is an Open Access article distributed in accordance with the Creative Commons Attribution-NonCommercial-NoDerivs 4.0 International License (CC BY-NC-ND 4.0), which permits the noncommercial replication and distribution of the article with the strict proviso that no changes or edits are made and the original work is properly cited (including links to both the formal publication through the relevant DOI and the license). See: https://creativecommons.org/ licenses/by-nc-nd/4.0/.

\section{References}

1. Uterine Sarcoma Treatment $(\mathrm{PDQ}(\mathrm{R}))$ : Health Professional Version. PDQ Cancer Information Summaries. Bethesda, MD: National Cancer Institute, 2002.

2. George S, Serrano C, Hensley ML, et al. Soft Tissue and Uterine Leiomyosarcoma. J Clin Oncol 2018;36:144-50.

3. Raine-Bennett T, Tucker LY, Zaritsky E, et al. Occult Uterine Sarcoma and Leiomyosarcoma: Incidence of and Survival Associated With Morcellation. Obstet Gynecol 2016;127:29-39. 
4. ACOG Committee Opinion No. 770: Uterine Morcellation for Presumed Leiomyomas. Obstet Gynecol 2019; 133:e238-48.

5. Graebe K, Garcia-Soto A, Aziz M, et al. Incidental power morcellation of malignancy: a retrospective cohort study. Gynecol Oncol 2015;136:274-7.

6. Oduyebo T, Rauh-Hain AJ, Meserve EE, et al. The value of re-exploration in patients with inadvertently morcellated uterine sarcoma. Gynecol Oncol 2014;132:360-5.

7. Senapati S, Tu FF, Magrina JF. Power morcellators: a review of current practice and assessment of risk. Am J Obstet Gynecol 2015;212:18-23.

8. Koh WJ, Abu-Rustum NR, Bean S, et al. Uterine Neoplasms, Version 1.2018, NCCN Clinical Practice Guidelines in Oncology. J Natl Compr Canc Netw 2018;16:170-99.

9. Giuntoli RL 2nd, Metzinger DS, DiMarco CS, et al. Retrospective review of 208 patients with leiomyosarcoma of the uterus: prognostic indicators, surgical management, and adjuvant therapy. Gynecol Oncol 2003;89:460-9.

10. Tasci T, Karalok A, Taskin S, et al. Does Lymphadenectomy Improve Survival in Uterine Leiomyosarcoma? Int J Gynecol Cancer 2015;25:1031-6.

11. Leitao MM, Sonoda Y, Brennan MF, et al. Incidence of lymph node and ovarian metastases in leiomyosarcoma of the uterus. Gynecol Oncol 2003;91:209-12.

12. Dindo D, Demartines N, Clavien PA. Classification of surgical complications: a new proposal with evaluation in a cohort of 6336 patients and results of a survey. Ann Surg 2004;240:205-13.

13. Food and Drug Administration. Quantitative assessment of the prevalence of unsuspected uterine sarcoma in women undergoing treatment of uterine fibroids: summary and key findings. Available online: https://www.fda.gov/ media/88703/download

14. Pedra Nobre S, Hensley ML, So M, et al. The impact of tumor fragmentation in patients with stage I uterine leiomyosarcoma on patterns of recurrence and oncologic outcome. Gynecol Oncol 2021;160:99-105.

15. Einstein MH, Barakat RR, Chi DS, et al. Management of uterine malignancy found incidentally after supracervical hysterectomy or uterine morcellation for presumed benign disease. Int J Gynecol Cancer 2008;18:1065-70.

16. Perri T, Korach J, Sadetzki S, et al. Uterine leiomyosarcoma: does the primary surgical procedure matter? Int J Gynecol Cancer 2009;19:257-60.

17. George S, Barysauskas C, Serrano C, et al. Retrospective cohort study evaluating the impact of intraperitoneal morcellation on outcomes of localized uterine

leiomyosarcoma. Cancer 2014;120:3154-8.

18. Xu X, Lin H, Wright JD, et al. Association Between Power Morcellation and Mortality in Women With Unexpected Uterine Cancer Undergoing Hysterectomy or Myomectomy. J Clin Oncol 2019;37:3412-24.

19. Bogani G, Cliby WA, Aletti GD. Impact of morcellation on survival outcomes of patients with unexpected uterine leiomyosarcoma: a systematic review and meta-analysis. Gynecol Oncol 2015;137:167-72.

20. Seagle BL, Sobecki-Rausch J, Strohl AE, et al. Prognosis and treatment of uterine leiomyosarcoma: A National Cancer Database study. Gynecol Oncol 2017;145:61-70.

21. Tokunaga H, Takahashi F, Yamamoto H, et al. Current Status of Uterine Leiomyosarcoma in the Tohoku Region: Results of the Tohoku Translational Center Development Network Survey. Int J Clin Oncol 2017;22:541-7.

22. Nasioudis D, Chapman-Davis E, Frey M, et al. Safety of ovarian preservation in premenopausal women with stage I uterine sarcoma. J Gynecol Oncol 2017;28:e46.

23. Seagle BL, Kanis M, Kocherginsky M, et al. Stage I uterine carcinosarcoma: Matched cohort analyses for lymphadenectomy, chemotherapy, and brachytherapy. Gynecol Oncol 2017;145:71-7.

24. Si M, Jia L, Song K, et al. Role of Lymphadenectomy for Uterine Sarcoma: A Meta-Analysis. Int J Gynecol Cancer 2017;27:109-16.

25. Tsikouras P, Dafopoulos A, Ammari A, et al. Should lymphadenectomy be performed in early stage I and II sarcomas of the corpus uteri. J Obstet Gynaecol Res 2011;37:1588-95.

26. Hoellen F, Waldmann A, Benthin S, et al. The role of lymphadenectomy in uterine sarcoma: a clinical practical approach based on retrospective analysis. Anticancer Res 2014;34:985-93.

27. Kim SI, Choi CH, Kim K, et al. Effectiveness of adjuvant treatment for morcellated, International Federation of Gynecology and Obstetrics stage I uterine leiomyosarcoma: A Korean multicenter study. J Obstet Gynaecol Res 2020;46:337-46.

28. Hensley ML, Wathen JK, Maki RG, et al. Adjuvant therapy for high-grade, uterus-limited leiomyosarcoma: results of a phase 2 trial (SARC 005). Cancer 2013;119:1555-61

29. Reed NS, Mangioni C, Malmström H, et al. Phase III randomised study to evaluate the role of adjuvant 
pelvic radiotherapy in the treatment of uterine sarcomas stages I and II: an European Organisation for Research and Treatment of Cancer Gynaecological Cancer Group Study (protocol 55874). Eur J Cancer
2008;44:808-18.

(English Language Editor: L. Huleatt)

Cite this article as: Yang J, Yang J, Cao D, Pan L, Wu M, Xiang Y. Management of stage I occult uterine leiomyosarcoma: effects of morcellation, second-look surgery, and adjuvant treatments on survival. Ann Transl Med 2022;10(2):127. doi: 10.21037/atm-21-6424 